Mar a Jun $2021-v .11-\mathrm{n} .2$

\title{
Características sensoriais de licor artesanal de umbu com microalga
}

O umbu (Spondias tuberosa Arruda) é uma fonte vegetal muito importante para a Caatinga, por possuir compostos importantes para alimentação. A microalga Spirulina spp. tem uso como suplemento alimentar funcional. No presente estudo, objetivou-se elaborar e avaliar as características sensoriais dos licores das folhas e dos frutos de umbu, acrescidos de microalga Spirulina spp., obtidos no Instituto Federal de Alagoas, localizado no município de Piranhas - AL. Foram elaboradas quatro formulações de licor da folha do umbu e quatro formulações de licor da polpa do umbu, adicionados de microalga Spirulina spp. Os licores foram avaliados sensorialmente quanto aos atributos aparência, cor, aroma, sabor e textura, utilizando-se da escala hedônica estruturada de nove pontos, sendo os extremos desgostei muitíssimo (1) e gostei muitíssimo (9). Para a intenção de compra, utilizou-se a escala estruturada de cinco pontos, cujos extremos foram eu certamente compraria (1) e eu certamente não compraria (5). A partir destes resultados, observou-se a capacidade destes licores no processamento agroindustrial, pois possuem elevados rendimentos. E que a inclusão de microalga potencializa o valor nutricional do licor, com elevada aceitação sensorial pelos potenciais consumidores.

Palavras-chave: Caatinga; Atributos; Valor Nutricional; Aceitação.

\section{Sensory characteristics of umbu handmade liquor with microalgae}

Umbu (Spondias tuberosa Arruda) is a very important plant source for Caatinga, because it has important compounds for feeding. The microalgae Spirulina spp. has use as a functional dietary supplement. In the present study, the objective of this study was to elaborate and evaluate the sensory characteristics of leaf liqueurs and umbu fruits, added to Spirulina spp., obtained at the Federal Institute of Alagoas, located in the municipality of Piranhas - AL. Four formulations of umbu leaf liquor and four umbu pulp liquor formulations were elaborated, added from microalgae Spirulina spp. The liqueurs were sensorially evaluated for the attributes appearance, color, aroma, flavor and texture, using the structured hedonic scale of nine points, being extremes very much disliked (1) and I liked it very much (9). For the purchase intention, the structured five point scale was used, whose extremes were: I would certainly buy (1) and I certainly would not buy (5). Based of these results, the capacity of these liquors in agro-industrial processing was observed, as they have high yields. And that the inclusion of microalgae enhances the nutritional value of the liquor, with high sensory acceptance by potential consumers.

Keywords: Caatinga; Attributes; Nutritional value; Acceptance.

Topic: Tecnologia de Alimentos

Reviewed anonymously in the process of blind peer.
Received: 10/03/2020

Approved: 30/03/2020
Vanessa Dayane Nunes da Silva (iD) Instituto Federal de Alagoas, Brasil http://lattes.cnpq.br/7736536164992730 http://orcid.org/0000-0001-8353-1517 nessinhadayane12@gmail.com

Victória Carolline do Moraes Gatti (iD) Universidade Federal Rural da Amazônia, Brasil http://lattes.cnpq.br/5799448994510549

http://orcid.org/0000-0002-7400-1685 victoriagatti.agro@gmail.com

Fábio Israel Martins Carvalho (id Universidade Federal Rural da Amazônia, Brasil http://lattes.cnpq.br/8221002637257793 http://orcid.org/0000-0002-8995-2141 fabioimc@yahoo.com.br
Job Teixeira de Oliveira (it)

Universidade Federal do Mato Grosso do Sul, Brasil http://lattes.cnpq.br/7250230926512123 http://orcid.org/0000-0001-9046-0382 job.oliveira@hotmail.com

Priscilla Andrade Silva (iD

Universidade Federal Rural da Amazônia, Brasi http://lattes.cnpq.br/7666887041806711 http://orcid.org/0000-0002-2774-3192 prisciandra@yahoo.com.br

Maria Cecília Castelo Branco de Santana (iD Instituto Federal de Alagoas, Brasil http://lattes.cnpq.br/9334405638611326 http://orcid.org/0000-0002-1482-0347 ceciliacastelob@gmail.com
João Paixão dos Santos Neto (iD Instituto Nacional de Investigação Agrária Veterinária de Portugal, Portugal http://lattes.cnpq.br/2828947517814190 http://orcid.org/0000-0003-4645-6866 joaopaixaoneto@gmail.com

\section{Referencing this:}

DOI: 10.6008/CBPC2237-9290.2021.002.0002

SILVA, V. D. N.; GATTI, V. C. M.; CARVALHO, F. I. M.; OLIVEIRA, J. T.; SILVA, P. A.; SANTANA, M. C. C. B.; NETO, J. P. S.. Características sensoriais de licor artesanal de umbu com microalga. Natural Resources, v.11, n.2, p.7-12, 2021. DOI: http://doi.org/10.6008/CBPC2237-9290.2021.002.0002 


\section{INTRODUÇÃO}

O nordeste brasileiro se caracterizou como um importante produtor de frutas tropicais. A fruticultura constitui-se como uma atividade econômica promissora em decorrência das condições climáticas prevalecentes e sua fruticultura é caracterizada por frutos aromáticos e de sabores exóticos (CAMPOS, 2007).

Dentre as diversas espécies presentes no Nordeste, o umbuzeiro se apresentou como uma importante espécie com potencial de exploração no semiárido brasileiro em decorrência de sua importância socioeconômica para as populações rurais (COSTA et al., 2015). Além do potencial econômico, o umbuzeiro se destaca como um grande provedor de vitamina c, seu valor nutricional é consideravelmente alto e possibilita a produção de diversos tipos de produtos alimentícios. 0 conhecimento do valor nutricional das frutas assume uma importância considerável, pois a alimentação adequada e a aplicação de métodos tecnológicos eficientes só se tornam possíveis mediante o conhecimento dos valores nutricionais (BASTOS et al., 2016).

Os licores são alternativos para o aproveitamento de frutas regionais, que agregam valor e possibilitam uma forma de gerar renda para famílias rurais (VIEIRA et al., 2010). No mercado, os licores estão ganhando espaço devido à diversidade de cores, viscosidade e sabores (SOUZA et al., 2018). Sobre a aceitabilidade, Teixeira et al. (2007) ressalta que a preferência por dado teor alcoólico de um licor está relacionada à combinação do gosto doce, o teor alcoólico e o sabor da fruta, observa-se esse fato em licores no mercado onde o teor muda de acordo com o sabor da fruta.

O objetivo deste estudo foi elaborar e caracterizar sensorialmente o licor funcional típico da Caatinga a base de folhas e frutos do umbuzeiro acrescidos de diferentes proporções de microalga Spirulina spp.

\section{METODOLOGIA}

As folhas e os frutos do umbuzeiro foram coletados no município de Piranhas, localizado na mesorregião do Sertão Alagoano, tem uma altitude aproximada de $88 \mathrm{~m}$ e coordenadas geográficas de $9^{\circ} 37^{\prime} 38^{\prime \prime}$ de latitude sul e $37^{\circ} 45^{\prime} 25^{\prime \prime}$ de longitude oeste. As folhas e os frutos foram selecionados em estádio maduros provenientes da safra 2018/19. A alga Spirulina spp. em pó foi adquirida em loja de manipulação, localizada em Maceió-Alagoas.

Foram elaboradas quatro formulações de licor da folha do umbu e quatro formulações de licor da polpa do umbu, adicionados de microalga Spirulina spp., de acordo com a Tabelas 1. Em cada tratamento, realizou-se a infusão através da maceração das folhas e a imersão dos frutos do umbu em cachaça de graduação alcoólica de 37,5\% (v/v) por 20 dias. Depois desse período, as soluções foram filtradas em filtro de nylon e os extratos alcoólicos obtidos foram misturados com xarope de sacarose a 43 oBrix. Adicionaram-se as concentrações de microalga Spirulina spp. em vasilhame tipo inox, previamente sanitizado. Após 60 dias de envelhecimento, realizaram-se a filtragem final dos licores, com o uso de uma bomba a vácuo em frasco tipo kitassato de 1 litro, acoplado por um funil tipo Büchner com filtro de 
celulose. Em seguida realizou-se a embalagem em garrafas de vidro e armazenadas em temperatura ambiente.

Tabela 1: Desenvolvimento dos licores com folhas e polpa de umbu com microalga Spirulina spp.

\begin{tabular}{|c|c|c|c|}
\hline \multicolumn{4}{|c|}{ Formulações dos licores com as folhas de umbu } \\
\hline Tratamento & Folhas do umbu (g) & Cachaça $(\mathrm{mL})$ & Spirulina spp. (g) \\
\hline T1 & 400 & 970 & 0,0 \\
\hline $\mathrm{T} 2$ & 400 & 970 & 0,3 \\
\hline T3 & 400 & 970 & 0,9 \\
\hline T4 & 400 & 970 & 1,5 \\
\hline \multicolumn{4}{|c|}{ Formulações dos licores com a polpa de umbu } \\
\hline Tratamento & Polpa de umbu (g) & Cachaça $(\mathrm{mL})$ & Spirulina spp. (g) \\
\hline T1 & 800 & 970 & 0,0 \\
\hline $\mathrm{T} 2$ & 800 & 970 & 0,3 \\
\hline T3 & 800 & 970 & 0,9 \\
\hline T4 & 800 & 970 & 1,5 \\
\hline
\end{tabular}

Os licores foram avaliados sensorialmente por 100 provadores, maiores de dezoito anos, quanto aos atributos aparência, cor, aroma, sabor e textura, utilizando-se da escala hedônica estruturada de nove pontos, sendo os extremos desgostei muitíssimo (1) e gostei muitíssimo (9). Para a intenção de compra, utilizou-se a escala estruturada de cinco pontos, cujos extremos foram: eu certamente compraria (1) e eu certamente não compraria (5) (DUTCOSKY et al., 2013). A análise estatística das variáveis respostas foi realizada com o auxílio do software Agroestat (BARBOSA et al., 2015). Após análise de variância as médias foram comparadas pelo Teste de Tukey, com $p \leq 0,05$.

\section{RESULTADOS E DISCUSSÃO}

Os licores das folhas e da polpa de umbu foram submetidos ao teste de aceitação sensorial por 100 provadores não treinados, as características sensoriais, podem ser observadas nas Figuras 1 e 2 .

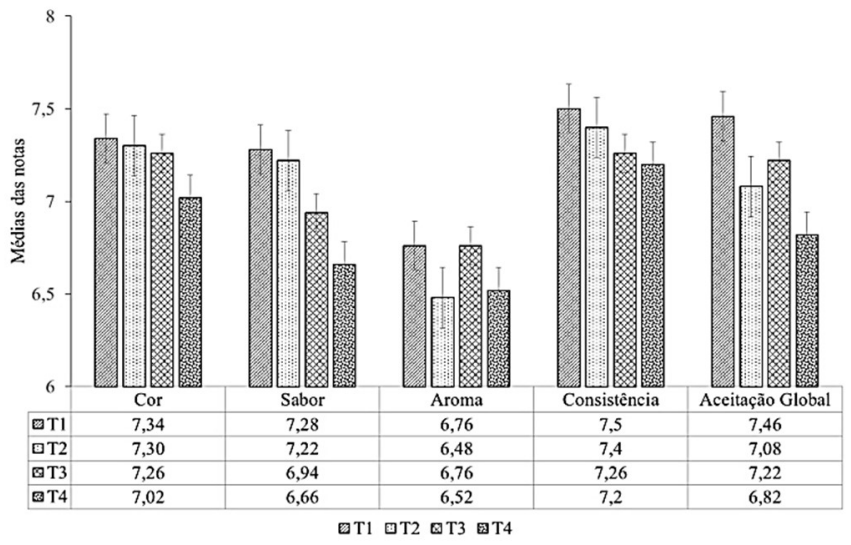

Figura 1: Análise sensorial dos licores de folhas de umbu com microalga Spirulina spp. T1 - Controle, T2 - 0,3\% de Spirulina spp., T3-0,9\% de Spirulina spp., T4 - 1,5\% de Spirulina spp.

A partir dos resultados, os provadores relatam que a cor obteve maiores médias nos tratamentos T1 e T2, (7,34 e 7,30), respectivamente. O sabor seguiu a mesma tendência da cor, sendo os tratamentos T1 e T2, melhor avaliados com valores médios atribuídos notas de 7,28 e 7,22, respectivamente. Um resultado com maior aceitabilidade que o encontrado por Muatiacale (2016) na pesquisa de licores de 
banana e de banana com anís que obteve média de 6,10 para a aparência e sabor. Esse resultado corrobora de forma positiva para uma resposta positiva do mercado.

O atributo aroma é o fator mais relevante na decisão de compra dos consumidores, porém os provadores julgaram que, tanto no tratamento T1, quanto no tratamento T3, não apresentaram diferença, ambos com média de 6,76. Shigematsu et al (2018), apresentou médias de 5,94 e 6,28 para aroma e consistência respectivamente com a análise de licores de abacaxi com coco e hortelã com pimenta. A consistência do licor de folhas de umbu, reflete a mesma tendência dos atributos de cor e sabor; com maior valor médio obtido para o tratamento $\mathrm{T} 1(7,50)$ seguido pelo tratamento $\mathrm{T} 2(7,40)$.

No sentido geral o tratamento T1 foi melhor avaliado pelos possíveis consumidores, com média de 7,46. A aceitação global dos licores apresentados por Shigematsu et al (2018) também apresentou uma média consideravelmente mais baixa, que foi de 4,56 .

Em relação a intenção de compra dos licores de folhas de umbu, Figura 2. Os dados indicam que o tratamento T1 foi o que obteve um percentual maior de aprovação, ou seja, foi o mais aceito com $54 \%$ dos provadores que afirmaram que 'certamente comprariam' o produto. Já no quesito que avalia a intenção de que o potencial consumidor 'provavelmente compraria' o produto, o tratamento que apresentou maior intenção foi o T3 com 44\%, esses índices de intenção de compra foram maiores que os índices encontrados por Souza et al. (2019) em sua pesquisa com o licor de abacaxi com hortelã e pimenta calabresa desidratada, que obteve em sua avaliação $46,7 \%$ que certamente comprariam e $43 \%$ provavelmente comprariam.

Almeida (2019), obteve uma melhor avaliação em relação a intenção de compra em sua análise de intenção de compra do licor de goiaba, sendo que $85 \%$ dos provadores demonstraram que certamente comprariam, o que consolida que o produto foi bem aceito e demonstra uma maior probabilidade de aceitação no mercado.

Por outro lado, o tratamento que obteve menor avaliação foi o T4, com $20 \%$ dos compradores que afirmaram que 'certamente não comprariam' o produto. No quesito que avalia a intenção de que o potencial consumidor 'provavelmente compraria' o produto, o tratamento que apresentou maior intenção foi o T3 com $44 \%$.

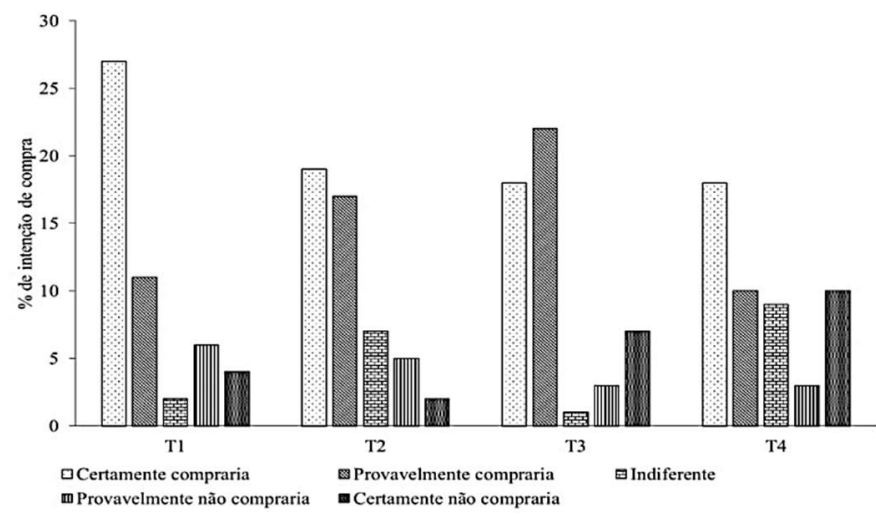

Figura 2: Intenção de compra dos licores de folhas de umbu com microalga Spirulina spp. T1 - Controle, T2 - 0,3\% de Spirulina spp., T3 - 0,9\% de Spirulina spp., T4 $-1,5 \%$ de Spirulina spp..

A partir dos resultados obtidos da análise sensorial dos licores da polpa de umbu, Figura 3, pode-se 
observar que a cor foi bem pontuada no tratamento $\mathrm{T} 2(7,20)$, seguida pelo tratamento $\mathrm{T} 3(7,14)$, isso implica que a Spirulina possivelmente contribuiu na composição do 'flavor' destes tratamentos. O parâmetro sabor foi bem avaliado no tratamento T1 $(7,4)$. Soares et al. (2017) com a análise de licores mistos de acerola e goiaba xaropado com mel, apontou um melhor desempenho do atributo cor, com o índice de 7,56 e de 6,79 para o sabor.

No tocante ao atributo aroma, a melhor formulação de acordo com os provadores foi a T3 $(6,7)$. A consistência revelou que os tratamentos T1 e T2 apresentaram as melhores médias $(7,2)$. No sentido geral de aceitação global o tratamento T3 $(7,02)$ foi melhor avaliado, seguido pelo tratamento T1 $(6,98)$. Em comparação, Soares et al. (2017) no melhor índice obtido na pesquisa de licor de caju, apresentou 7,50 para o atributo aroma, já para o aspecto global o índice indicado foi de 7,29 próximo ao indicado pelo aspecto global do licor da polpa de umbu.

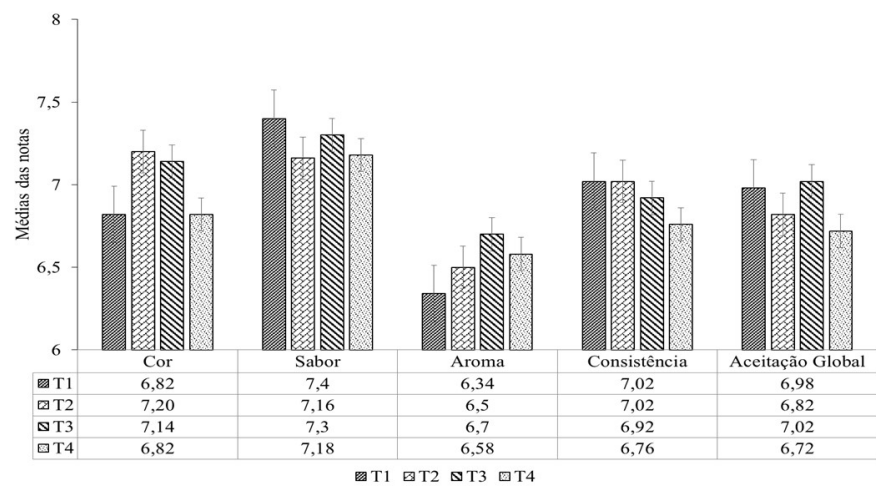

Figura 3: Análise sensorial dos licores da polpa de umbu com microalga Spirulina spp. T1 - Controle, T2 - 0,3\% de Spirulina spp., T3 - 0,9\% de Spirulina spp., T4 - 1,5\% de Spirulina spp.

A intenção de compra das diferentes formulações de licor de polpa de umbu está disposta na Figura 4. Paulatinamente, os tratamentos com maiores intenção de compra foi o T4 com $38 \%$ dos potenciais consumidores, seguidos pelos tratamentos T1 e T3, com 36 e $34 \%$ dos provadores que afirmaram que 'certamente comprariam' os produtos. Ainda que os índices de T4, T1 E T3 estejam em conformidade e não tenham uma variância muito elevada, em comparação ao trabalho de Almeida (2019), que obteve um índice de intenção de compra mais elevado, Oliveira et al. (2011), também apresentou uma melhor intenção de compra em seu trabalho com licor de açaí, com $74,8 \%$ dos provadores assinalando que provavelmente ou certamente comprariam.

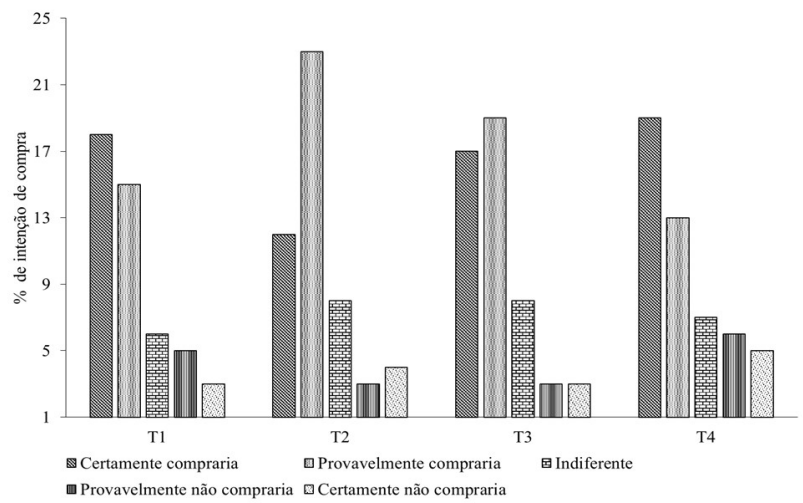

Figura 4: Intenção de compra dos licores de polpa de umbu com microalga Spirulina spp. T1 - Controle, T2 - 0,3\% de Spirulina spp., T3-0,9\% de Spirulina spp., T4 - 1,5\% de Spirulina spp. 
A Figura 4 também indica que o tratamento T2 foi o que obteve maior percentual de intenção de compra e os provadores julgaram que 'provavelmente comprariam' o licor de polpa de umbu com 0,3\% de microalga Spirulina spp.

\section{CONCLUSÃO}

As partir da aplicação da análise sensorial, os licores das folhas e dos frutos de umbu, com adição de diferentes porcentagens de microalga Spirulina spp., revelaram ser bem aceitos, com elevada aceitação global. Realizar o estudo sensorial desses produtos obtidos é fundamental para seus usos na indústria de alimentos como na alimentação humana.

Sendo assim, a execução do estudo proposto promoveu avanços no conhecimento sobre o processo agroindustrial de licores artesanais com produtos típicos do Sertão Nordestino. Recomendam-se estudos futuros, na mesma linha, com outros frutos típicos da Caatinga.

\section{REFERÊNCIAS}

ALMEIDA, J. C.. Elaboração, caracterização físico-química e aceitabilidade de licor de goiaba. Instituto Federal Goiano, 2019.

BARBOSA, J. C.; MALDONADO, J. W.. AgroEstat: sistema para análises estatísticas de ensaios agronômicos. Jaboticabal: FCAV/UNESP, 2015.

BASTOS, J. S.; MARTINEZ, E. A.; SOUZA, S. M. A.. Características físico-químicas da polpa de umbu (Spondias tuberosa Arruda Câmara) comercial: efeito da concentração, Journal of Bioenergy and Food Science, v.3, n.1, p.11-16, 2016.

CAMPOS, C. O.. Frutos de umbuzeiro (Spondias tuberosa): características físico-químicas durante seu desenvolvimento e na pós-colheita. Botucatu: UNESP, 2007.

COSTA, F. R.; RÊGO, E. R.; RÊGO, M. M.; NEDER, D. G.; SILVA, S. M.; SCHUNEMANN, A. P. P.. Análise biométrica de frutos de umbuzeiro do Semiárido Brasileiro. Bioscience Journal, v.31, n.3, p.682-690, 2015.

DUTCOSKY, S. D.. Análise sensorial de alimentos. 4 ed. Curitiba: Champagnat, 2013.

MINIM, V. P. R.. Análise sensorial: estudos com consumidores. 3 ed. Viçosa: EdUFV, 2013.

MUATIACALE, T. A. A. S.. Elaboração e avaliação sensorial de licores caseiros de banana e de banana com anís.
Monografia (Licenciatura em Enganharia Alimentar) Universidade Católica de Moçambique, Chimoio, 2016.

OLIVEIRA, E. N. A.; SANTOS, D. C.. Processamento e avaliação da qualidade de licor de açaí (Euterpe oleracea mart.), Revista do Instituto Adolfo Lutz, v.70, n.4, 2011.

SHIGEMATSU, E.; DORTA, C.; MARINELLI, P. S.; OSHIIWA, M.; GOLIM, J. F.; SANTOS, M. A. F.; ROCATTO, I. G..Determinação do tempo de maturação dos licores de abacaxi com coco e hortelã com pimenta: análises sensoriais e físico-químicas. Unimar Ciências, v.27, n.1-2, 2018.

SOARES, A. P.; OLIVEIRA, E. N. A.; FEITOSA, R. M.; SILVA, R. C.; ARAÚJO, K. T. A.. Perfil sensorial e aceitação de licores mistos de acerola e goiaba xaropado com mel. Revista Brasileira de Agrotecnologia, v.7, n.2, p.126-131, 2017.

SOUZA, L. R.; DIAS, J. V.; SOUZA, S. A. N.; SILVA, L. K. B.; SILVA, B. A.; JOELE, M. R. S. P.. Caracterização físico-química e sensorial de licor de abacaxi com hortelã e pimenta calabresa desidratada. Atena, p.143-149, 2018.

TEIXEIRA, L. J. Q.; RAMOS, A. M.; CHAVES, J. B. P.; STRINGHETA, P. C.. Testes de aceitabilidade de licores de banana. Revista Brasileira Agrociência, v.13, n.2, p.205-209, 2007.

VIERA, V. B.; RODRIGUES, J. B.; BRASIL, C. C. B.; ROSA, C. S.. Produção, caracterização e aceitabilidade de licor de camucamu (myrciaria dúbia (h.B.K.) Mcvaugh). Alimentos e Nutrição, v.21, n.4, p.520, 2010.

A CBPC - Companhia Brasileira de Produção Científica (CNPJ: 11.221.422/0001-03) detém os direitos materiais desta publicação. Os direitos referem-se à publicação do trabalho em qualquer parte do mundo, incluindo os direitos às renovações, expansões e disseminações da contribuição, bem como outros direitos subsidiários. Todos os trabalhos publicados eletronicamente poderão posteriormente ser publicados em coletâneas impressas sob coordenação da Sustenere Publishing, da Companhia Brasileira de Produção Científica e seus parceiros autorizados. Os (as) autores (as)

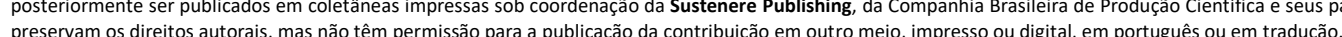

